\title{
Effect of Resonator Axis Skew on Normal Incidence Impedance
}

\author{
Tony L. Parrott, ${ }^{\prime M}$ Michael G. Jones, ${ }^{\ddagger}$ Brian Homeijer ${ }^{\S}$ \\ NASA Langley Research Center, Hampton, VA
}

\begin{abstract}
$\underline{\text { Abstract }}$
High by-pass turbofan engines have fewer fan blades and lower rotation speeds than their predecessors. Consequently, the noise suppression at the low frequency end of the noise spectra has become an increasing concern. This has led to a renewed emphasis on improving noise suppression efficiency of passive, duct liner treatments at the lower frequencies. For a variety of reasons, passive liners are comprised of locally-reacting, resonant absorbers. One reason for this design choice is to satisfy operational and economic requirements. The simplest liner design consists of a single layer of honeycomb core sandwiched between a porous facesheet and an impervious backing plate. These resonant absorbing structures are integrated into the nacelle wall and are very efficient over a limited bandwidth centered on their resonance frequency. Increased noise suppression bandwidth and greater suppression at lower frequencies is typically achieved for conventional liners by increasing the liner depth and incorporating thin porous septa into the honeycomb core. However, constraints on liner depth in modern high by-pass engine nacelles severely limit the suppression bandwidth extension to lower frequencies. Also, current honeycomb core liners may not be suitable for irregular geometric volumes heretofore not considered.

It is of interest, therefore, to find ways to circumvent liner depth restrictions and resonator cavity shape constraints. One way to increase effective liner depth is to skew the honeycomb core axis relative to the porous facesheet surface. Other possibilities are to alter resonator cavity shape, e.g. high aspect ratio, narrow channels that possibly include right angle bends, $180^{\circ}$ channel fold-backs, and splayed channel walls to conform to irregular geometric constraints. These possibilities constitute the practical motivation for expanding impedance modeling capability to include unconventional resonator orientations and shapes.
\end{abstract}

\footnotetext{
$\dagger$ Senior Research Scientist, Structural Acoustics Branch, Aerodynamics, Aerothermodynamics and Acoustics Competency

${ }^{\ddagger}$ Research Scientist, Structural Acoustics Branch, Aerodynamics, Aerothermodynamics and Acoustics Competency, Member of AIAA

${ }^{\S}$ LARSS Student, Lehigh University, Bethlehem, PA 18015
}

The work reported in this paper is in the nature of a progress report and is limited to examining the implications of resonator axis skew on the composite normal incidence impedance of an array of resonator channels. Specifically, experimental results are compared with a modified impedance prediction model for highaspect-ratio, rectangular, resonator channels with varying amounts of skew relative to the incident particle velocity. It is shown that for resonator channel widths of 1 to $2 \mathrm{~mm}$, aspect ratios of 25 to 50 , and skew angles of zero to sixty degrees, the surface impedance of test models can be predicted with good accuracy. Predicted resistances and reactances are particularly well correlated through the first resonance and first anti-resonance for all six test models investigated. Beyond the first anti-resonance, the impedance prediction model loses the ability to predict details of resistance and reactance but still predicts the mean trends very well.

\section{$\underline{\text { Nomenclature }}$}

\begin{tabular}{|c|c|}
\hline$c$ & sound speed, $\mathrm{m} / \mathrm{s}$ \\
\hline$h$ & depth of honeycomb core, $\mathrm{mm}$ \\
\hline$L$ & channel length, $\mathrm{mm}$ \\
\hline$R_{a}$ & aspect ratio \\
\hline \multicolumn{2}{|c|}{ Symbols: } \\
\hline $\bar{\phi}$ & channel skew angle, degrees \\
\hline$\rho$ & ambient density, $\mathrm{kg} / \mathrm{m}^{3}$ \\
\hline$\theta$ & $\begin{array}{l}\text { normalized acoustic resistance, real com- } \\
\text { ponent of } \zeta\end{array}$ \\
\hline$\chi$ & $\begin{array}{l}\text { normalized acoustic reactance, imaginary } \\
\text { component of } \zeta\end{array}$ \\
\hline$\zeta$ & $\begin{array}{l}\theta+i \chi, \text { normal incidence acoustic } \\
\text { impedance, normalized by } \rho c\end{array}$ \\
\hline \multicolumn{2}{|c|}{ Abbreviations: } \\
\hline $\mathrm{cc}$ & circular channel \\
\hline LaRC & Langley Research Center \\
\hline SLA & steriolithography apparatus \\
\hline SPL & sound pressure level, $\mathrm{dB}$ \\
\hline TMM & Two-Microphone Method \\
\hline ZKTL & Zwikker-Kosten Transmission Line code \\
\hline
\end{tabular}

An $e^{i \omega t}$ time convention is used throughout this paper. 


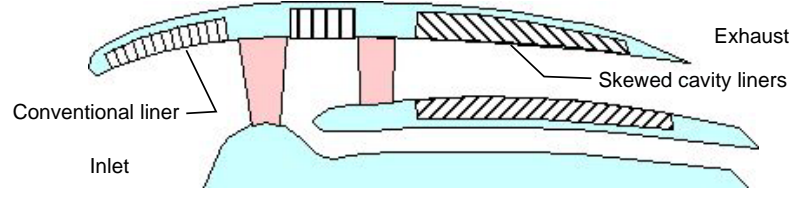

Figure 1. Nacelle liner installation.

\section{Introduction}

Although passive, absorptive liner technology has reached a relatively mature state, it remains a critical component in the overall noise abatement methodology for commercial aircraft engines. Further improvements and innovative applications for passive liner technology are likely in the future because of new developments in materials science, fabrication processes, and computational modeling. It is these enabling technologies that provide the impetus for the project described in this paper.

The advent of high by-pass turbofan engines has stimulated the search for and development of innovative liner concepts. These engines generally produce more noise than their predecessors in the low frequency part of the noise spectrum that relates to community annoyance. ${ }^{1}$ The broadband contribution from wide chord fans makes this noise less dominated by discrete frequencies. To complicate matters further, the high by-pass engine nacelle can accomodate less liner length relative to the nacelle diameter, especially in the fan inlet, as depicted in figure 1. Also, the nacelle wall thickness (which translates into depth available for liners) does not increase proportionately with increasing fan diameter. This evolving engine-cycle trend requires increasing liner efficiency just to maintain the same noise reduction obtainable with the lower by-pass engines of the last century. One way to cope with liner depth constraint is to distort, or otherwise modify, the conventional honeycomb core to lower the resonance frequency and thus enhance low frequency absorption efficiency.

The semi-empirical character of current impedance prediction models may limit the impedance prediction accuracy of liner structures that depart from the conventional honeycomb-core architectures. The reason for this is that the conventional honeycomb-core liner is typically modeled as a one-dimensional, non-dissipative acoustic system. The honeycomb core contribution to the liner impedance is purely reactive. The facesheet contributes nearly all of the resistance, as well as a mass-like reactive component that increases linearly with frequency. This model is incapable of accounting for the liner behavior in a relatively small frequency range centered on anti-resonance frequencies. Thus, for a complex liner
Conventional liner (honeycomb core)

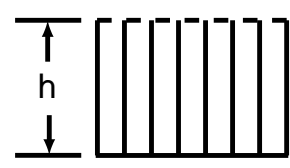

$\mathrm{h}(\cos \phi)=0.71 \mathrm{~h}$ at $\phi=45^{\circ}$

Modified liner (skewed resonator channels)

-> $30 \%$ reduction in depth for no reduction in resonance frequency

Figure 2. Conventional and skewed resonator channels.

geometry where dissipation may be present in the resonator channels and a composite impedance is desired for a group of contiguous resonators with channel-tochannel depth variations, this simple model may lose accuracy. Yet, the accuracy of impedance prediction models for such structures is crucial to the achievement of target impedance spectra. It is therefore of some importance that the impedance contribution from unconventional resonator cavity architectures be accurately modeled. The simplest departure from conventional resonator geometry is a skewing of the channel axis relative to the porous surface normal. A simple example of this is illustrated in figure 2. In the sketch on the left, a conventional honeycomb core of depth $h$, with a thin resistive facesheet, is shown. In the sketch on the right, the resonator channels are skewed by the angle $\phi=45^{\circ}$, resulting in a depth reduction of about $30 \%$, but no change in resonance frequency. In some applications, not indicated in the sketch, channel-to-channel depth variations and splayed channel walls may be necesary to exploit irregularly shaped, available space. Depending upon the wavelength, the resonances of splayed wall resonators may be shape dependent if non-Helmholtz resonances are of concern. It is imperative that these effects be understood if the desired optimum impedance spectrum incorporating such unconventional resonator geometry is to be achieved.

This paper is a progress report on the first phase of an investigation that focuses on the effects of resonator axis skew on normal incidence impedance of high aspect ratio rectangular channels. The skew is measured relative to the resonator axis when it is coincident with the incident sound particle velocity at the resonator face. To achieve a measurable resistance without installation of a thin resistive facesheet in the plane of the resonator face, an array of rectangular channels is used with channel airway widths ranging from $1 \mathrm{~mm}$ to $2 \mathrm{~mm}$. Thus, the resistive component of the impedance is supplied by cavity wall dissipation. Although resonator channel skew is the main focus of this phase of the investigation, it was 


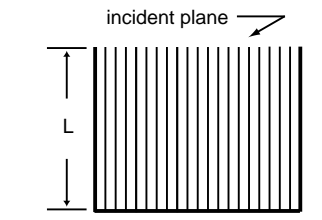

(a) Configuration $\mathrm{C} 1$; Straight section

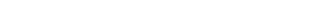

(1)

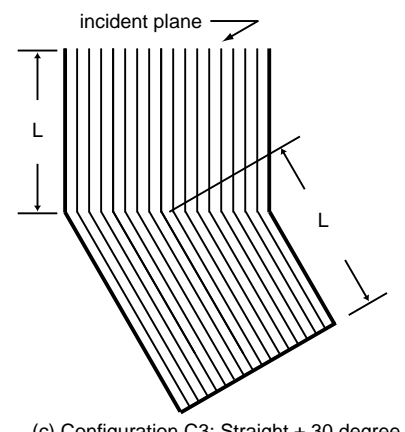

(c) Configuration C3; Straight +30 degree

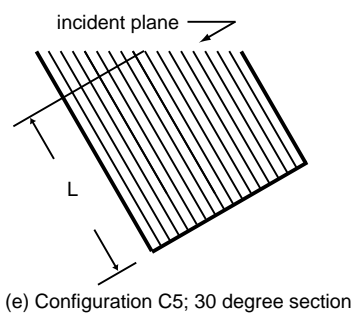

Figure 3. Sketch of test models.

necessary for the predictive model to also account for high-aspect-ratio rectangular channels and for channelto-channel depth variation. The necessity of including channel-to-channel depth variation was driven by test model fabrication convenience as will be described forthwith. Effects of cavity wall splay will be investigated in a later phase of this project.

\section{Test Model Fabrication and Design}

The test models were fabricated by means of a steriolithography apparatus (SLA) that works to an absolute precision of $\pm 0.0635 \mathrm{~mm}$. The apparatus provides for a numerically-positioned laser beam to map out, in successive layers, the prescribed solid portions of the test model in a fluid resin bath. The precision exposure of the fluid resin to the laser beam causes a layer-by-layer fabrication of the test model. A great advantage of this technology is that it allows fabrication of intricate, unitary structures that require no assemblage of machined parts.
Six test models were fabricated to study the effects of channel skew on the normal incidence impedance of arrays of contiguous resonators in the form of rigidly terminated rectangular channels. The resonator incident planes are co-planar with the $51 \mathrm{~mm}$-square impedance measurement plane of the NASA Langley Research Center (LaRC) Vertical Impedance Tube. The design philosophy for the test models will be discussed with the aid of the side-view sketches of figure 3 and the photograph of figure 4. The sketch sequence in figure 3 also represents the testing sequence. The straight channel test models illustrated in the sketches of figure $3-a, b$ are the baseline test cases which are used, in part, to validate the high aspect ratio modification of the impedance prediction model discussed in the "Impedance Prediction Model" section. The effect of channel skew was first studied with the aid of the two test models shown in figure 3-c,d. These test models are designed to compare effects of skewing half the length $(L=85.7 \mathrm{~mm})$ of the straight channel array (of length $2 \mathrm{~L}$ ) about the centerspan point by 30 and 60 degrees to generate test models respectively. (Note that these test models have 17 and 10 channels for 30 and 60 degrees of skew respectively.) This causes the straight and skewed sections to interface in a mitered joint, shortening and lengthening the channels on either side of the pivot point in a symmetrical manner. Thus, the averaged length of the skewed channels, taken together, does not change with skew. Also, the length of the central channel (or partition, as the case may be) is invariant. In this manner, the test models of figure 3-b,c,d are designed to isolate the skew effect from possible "contamination" by the classical "end effect" associated with the area discontinuity at the incident plane. The test models (figure 3-a,e,f) can be thought of as a replication of the test models (figure 3-b,c,d) with the upper straight section (of length $L$ ) removed. These test models are designed to provide a validation of the prediction model when end effects and skew effects coalesce at the incident plane.

From a fluid dynamic viewpoint, the effect of skew is to turn the normal incident particle velocity through the angle of skew, $\phi$. It is easy to visualize that when this occurs at the mitered joint of the straight and skewed sections of the test models in figure 3-c,d above, then the particle velocity in the skewed section must increase by the factor $1 / \cos \phi$ because of the corresponding reduction in channel width. Intuitively, one would expect a particle velocity increase to cause an increase in acoustic resistance contributed by the skewed section. The test models that combine straight and skewed channels through the mitered interface are designed to provide the simplest posssible validation of the skew effect because the skew effect is separated geometrically from the end effect at the incident plane. 
An important design requirement for the test models was to achieve a measurable resistance using the NASA LaRC Two-Microphone Method (TMM) impedance measurement methodology. This methodology achieves its maximum accuracy over a resistance range of about $0.25 \rho c$ to $10 \rho c$ and over a frequency range of $0.5 \mathrm{kHz}$ to $3.0 \mathrm{kHz}$. This measurement envelop, together with the fabrication precision limit indicated above, drove the baseline test models (straight channel sections in figure $3-a, b$ to have 20 channels with the following parameters: channel airway width, $1 \mathrm{~mm}$; channel depth, $85.7 \mathrm{~mm}$; and channel partition thickness, $1.63 \mathrm{~mm}$ (the minimum channel partition thickness).

The airway width of all the skew sections (figure 3$\mathrm{c}, \mathrm{d}, \mathrm{e}, \mathrm{f})$ was maintained at $1 \mathrm{~mm}$ with a fabrication precision of $\pm 0.0635 \mathrm{~mm}$. However, the number of channels in the 30 and 60 degree skew section (both when joined with a straight section and stand alone) was proportional to the baseline test model cross-section area projected through 30 and 60 degrees, respectively. This approximated to 17 and 10 channels in the 30 and 60 degree skewed sections, respectively. Once these geometric design choices are made for the skew sections, the channel airway and partition widths are determined for the associated straight continuation. For the straight section continuation from the 30 degree skew section, the straight channel airway width is $1.14 \mathrm{~mm}$ and the partition width is $1.96 \mathrm{~mm}$. For the straight section continuation from the 60 degree skew section, the airway width is $1.98 \mathrm{~mm}$ and the partition width is $3.45 \mathrm{~mm}$. All test model channel arrays were terminated by a $13 \mathrm{~mm}$-thick end-cap with a thin film of $0.08 \mathrm{~mm}$ Mylar sandwiched between the end-cap and channel array termination to serve as a sealing gasket between channels. It is believed that this arrangement provided for very nearly zero acoustic particle velocity at each channel termination and at least $20 \mathrm{~dB}$ of acoustic isolation between channels. All channels had the same span-wise dimension of $51 \mathrm{~mm}$ to give aspect ratios (ratio of channel span-wise dimension to channel airway width) ranging from about 25 to 50 .

\section{Impedance Prediction Model}

The set of test models described above were designed to validate an impedance prediction model for smallchannel width, high aspect ratio, rectangular channels that included channel axis skew with respect to the incident wave vector. The test model design parameters were chosen to exploit the existing experience with the NASA LaRC Zwikker-Kosten Transmission Line (ZKTL) propagation code for small diameter tubes. This code is described in previous reports. ${ }^{2,3}$ The code is based on a summary by Tijdeman ${ }^{4}$ of the classical work initiated in the latter part of the 18th century concerning sound propagation in small diameter tubes. The ZKTL-based

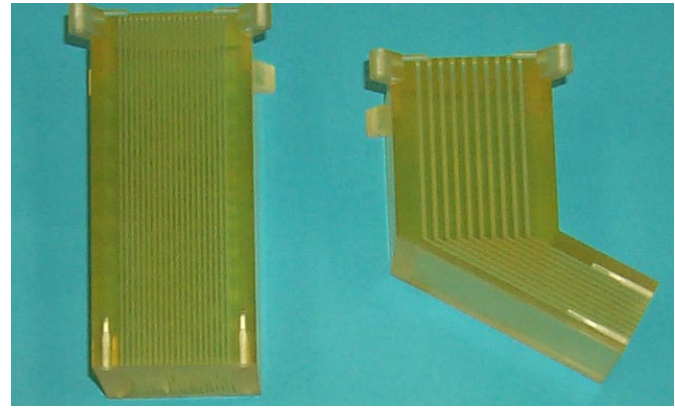

Figure 4. Photograph of straight \& skewed channel test models.

impedance prediction code ${ }^{3}$ is empirically corrected for rectangular channels with aspect ratios of approximately 9 by multiplying the normalized resistance component by the ratio $\left(R_{a}+1\right) / 2$; i.e.,

$$
\zeta_{\mathrm{cc}}=\frac{R_{a}+1}{2} \theta_{\mathrm{cc}}+i \chi_{\mathrm{cc}}
$$

where $R_{a}$ is the aspect ratio, and the subscript "cc" indicates quantities computed using the smallest crosssection dimension as an equivalent circular channel diameter.

Physically, the Zwikker-Kosten analysis allows one to calculate, in closed form, the propagation constant and characteristic impedance of a small diameter channel in which the acoustic boundary layer dominates the particle velocity profile. This causes significant dissipation of the acoustic wave to ensue. In practice, this happens for air-filled channels if the "effective" tube diameter is on the order of $1 \mathrm{~mm}$ or less. Thus, the present SLA fabricated test models meet that requirement wihout excessively compromising channel width precision.

At NASA LaRC, the ZKTL impedance prediction model has been validated for arrays of parallel tubes with "equivalent" diameters on the order of $1 \mathrm{~mm}$. In the present case with aspect ratios of 25 to 50, a generalized radius is used based upon the analysis of Richards. ${ }^{5}$ This analysis indicated that propagation through non-circular channels with high aspect ratio cross-sections can be adequately described by the Zwikker-Kosten analysis if a generalized tube radius is employed. This generalized radius is given by twice the channel cross-section area divided by the cross-section perimeter. For a rectangular channel, the generalized radius is the channel cross section area divided by the cross section perimeter. This generalized radius modification is incorporated into the the NASA LaRC ZKTL impedance prediction model for high aspect ratio rectangular channels discussed in this paper. 


\section{Vertical Impedance Tube Apparatus}

Impedance measurements were conducted in the NASA LaRC Vertical Impedance Tube apparatus (VIT). The essential features of the apparatus are depicted in figure 5. The vertical orientation is convenient for measuring the impedance of unconsolidated granular bulk materials that would otherwise require restraining to avoid spilling out into the impedance tube. The impedance measurement methodology at NASA LaRC is a version of the switchable two-microphone-method (TMM) that employs a rotating plug to switch the microphones and thus "average out" differences between the data acquisition channels. This circumvents a tedious calibraton procedure, the details of which are explained elsewhere. ${ }^{6,7}$ The methodology relies upon both the incident and reflected waves to be devoid of any measurable contaminanton from higher-order mode content at the measurement microphone locations (i.e. plane waves only). This requirement implies that any reflected wave nonuniformity generated at the test surface must have no measurable influence on the total wave field at the measurement microphone locations.

The impedance measurement plane for the VIT is located at its exit (see figure 5) and the test specimen surface is made coincident with this plane. A reference microphone is located about $6.35 \mathrm{~mm}$ from this plane and provides a measure of the total sound field at the test specimen surface. Figure 6 shows a photograph of the VIT with one of the present test models (3-c) mounted. Note that the transparancy of the apparatus allows the test model surface and environs to be viewed. This feature is regarded as valuable for including oscillatory flowvisualization should the need arise.

\section{Discussion of Results}

Figures 7 through 12 show comparisons of impedance spectra measurements and predicted values for the six test models. All measurements were conducted with bandwidth limited white noise over the frequency range of 0.5 to $3.5 \mathrm{kHz}$, in increments of $25 \mathrm{~Hz}$. The overall SPL (measured over frequency range of 0.5 to $3.0 \mathrm{kHz}$ ) was set to $140 \mathrm{~dB}$ at the reference microphone location. The impedance model predictions are calculated at $100 \mathrm{~Hz}$ intervals.

Figures 7 and 8 are the results for the straight, 20channel, test models of length $L$ and $2 L$, respectively. The dimension $L$ was set to $85.7 \mathrm{~mm}$ so that at least one anti-resonance is well defined for the shortest test model. That is clearly the case for configuration $\mathrm{C} 1$ in figure 7 where the first resonance at about $1.0 \mathrm{kHz}$ and a second resonance at about $3.0 \mathrm{kHz}$ bracket the anti-resonance at about $2.0 \mathrm{kHz}$. On the scale of this graph, resistance values of a few tenths of one $\rho c$ are not resolved well in

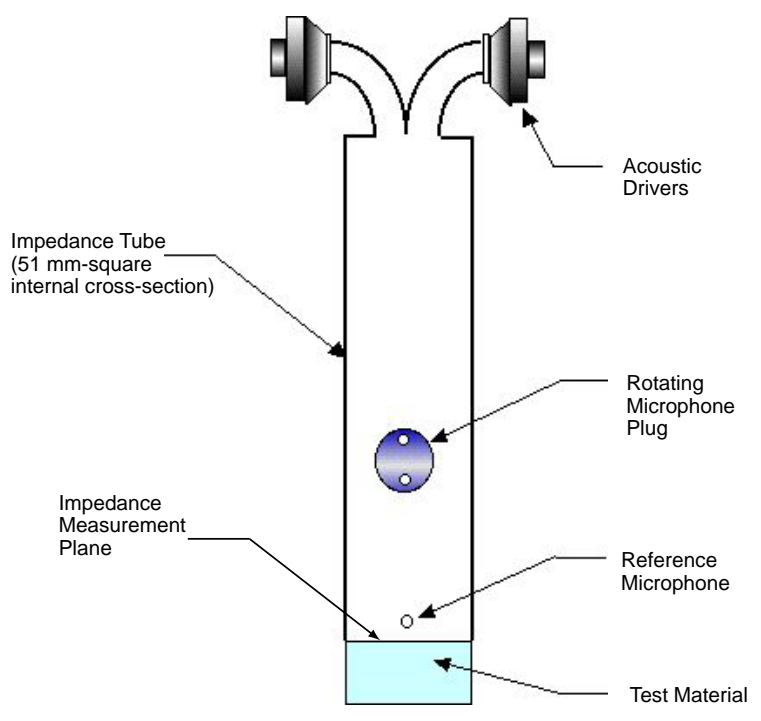

Figure 5. Vertically oriented impedance tube (VIT).

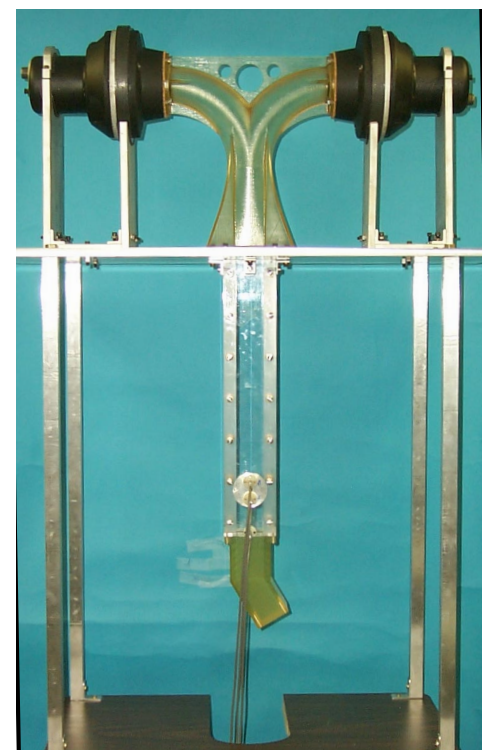

Figure 6. Photograph of Vertical Impedance Tube. 
the vicinity or resonance. Note, however, that predicted values of both resistance and reactance match the measured values very well throughout the frequency range of 0.5 to $3.0 \mathrm{kHz}$. For the straight, 20-channel, $2 L$ length test model (configuration C2), the results are shown in figure 8 and the same remarks generally apply as for figure 7 . In this case, there are 3 resonances $(0.5,1.5$ and $2.5 \mathrm{kHz}$ ) and 3 anti-resonances in the frequency range of 0.5 to $3.0 \mathrm{kHz}$. Measured and predicted impedances are again in excellent agreement. These results validate the impedance prediction model for contiguous, highaspect-ratio, rectangular channel test models.

Figure 9 shows results when a skew angle of 30 degrees is introduced into the 17-channel test model of length $2 L$ (sketch 3-c, configuration C3). This figure should be compared directly with figure 8 . Generally, both predicted resistance and reactance are in excellent agreement with measured results. Note that there is a significant decrease (but well predicted) in the measured values of resistance at the third anti-resonance for the 30 degree skew test model. The additional secondary peak in predicted resistance at about $3.3 \mathrm{kHz}$ was not validated by measurments in the $51 \mathrm{~mm}$-square impedance tube, since the maximum measurement frequency was $3.0 \mathrm{kHz}$.

Figure 10 shows results when a skew angle of 60 degrees is introduced into the $2 L$ length straight section (sketch 3-d, configuration C4). This figure should be compared directly with figure 8. Again, the measured and predicted impedance values are in very good agreement. There are significant changes generated by the introduction of 60 degrees of skew, and the prediction model does an excellent job of predicting the general nature of these changes. For instance, the first resonance moves from $500 \mathrm{~Hz}$ to about $600 \mathrm{~Hz}$ for both prediction and measurement. In addition, the first anti-resonance resistance values are reduced for both prediction and measurement. It is interesting to note that the third antiresonance essentially disappears with the addition of 60 degrees of skew, a prediction that is validated by the measurement.

Figures 11 and 12 show predicted and measured values of impedance for the the two test models without leadin straight sections, i.e. the incident sound is directly incident on the skewed channels. These figures should be compared directly with figure 7 . Here again, predicted and measured impedance values are in excellent agreement. Note the good agreement between the predicted and measured resistances at the peak of the antiresonance at about $20 \rho c$ for the 30 degrees of skew and about $27 \rho c$ for 60 degrees of skew.

\section{Concluding Remarks}

The following observations are drawn from the current investigation:

1. Generally, measured and predicted impedance values are in excellent agreement for both straight and skewed-channel test models.

2. The prediction model fails to resolve details of measured resistance variability at the high frequencies.

3. The results clearly show that resonator channel aspect ratio and skew factors have an important effect on on the nomal incidence impedance. Furthermore, the influence of these parameters can be adequately modeled by simple modifications to a wellknown model for propagation through small diameter, circular tubes. The aspect ratio factor is included by constructing a generalized radius equal to twice the channel cross section area divided by the channel perimeter. The angle of skew effect can be equated to a decrease in the channel open area ratio by the factor $1 / \cos \phi$.

\section{References}

1. Bielak, G. W., Premo, J. W., and Hersh, A. S., "Advanced Turbofan Duct Liner Concepts," NASA CR1999-209002, 1999.

2. Parrott, T. L., Watson, W. R., and Jones, M. G., "Experimental Validation of a Two-Dimensional ShearFlow Model for Determining Acoustic Impedance," NASA TP-2679, 1987.

3. Jones, M.G., "An Improved Model for ParallelElement Liner Impedance Prediction," AIAA 971649, 1997.

4. Tijdeman, H., "On the Propagation of Sound Waves in Cylindrical Tubes," Journal of Sound and Vibration, Vol. 39, No. 1, 1975, pp. 1-33.

5. Richards, W. B., "Propagation of Sound Waves in Tubes of Noncircular Cross Section," NASA TP 2601, 1986.

6. Jones, M. G., and Stiede, P. E., "Comparison of Methods for Determining Specific Acoustic Impedance," Journal of the Acoustical Society of America, Vol. 101, No. 5, 1997, pp. 2694-2704.

7. "Standard Test Method for Impedance and Absorption of Acoustical Materials Using a Tube, Two Microphones, and a Digital Frequency Analysis System," ASTM E1050-90, 1990. 


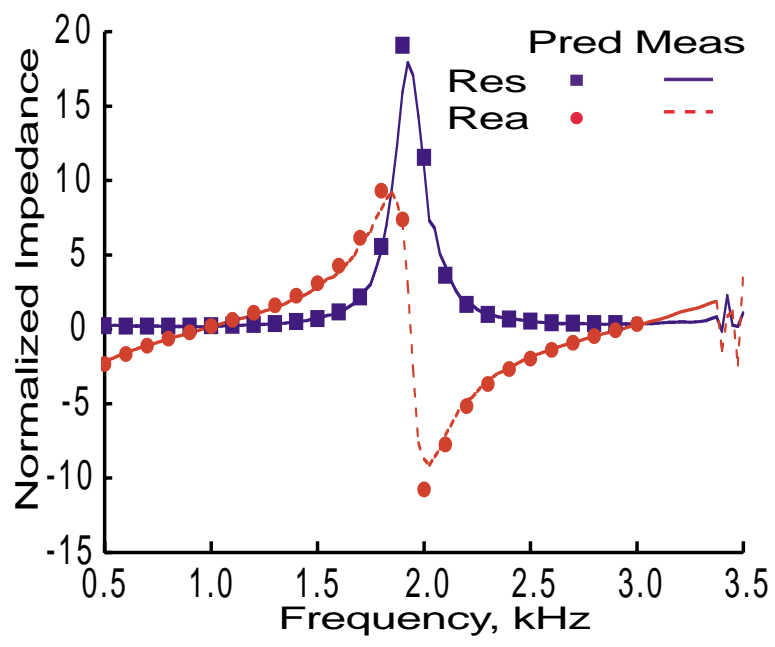

Figure 7. Normalized impedance for Configuration C1 (straight 20-channel baseline - length, $L$ ).

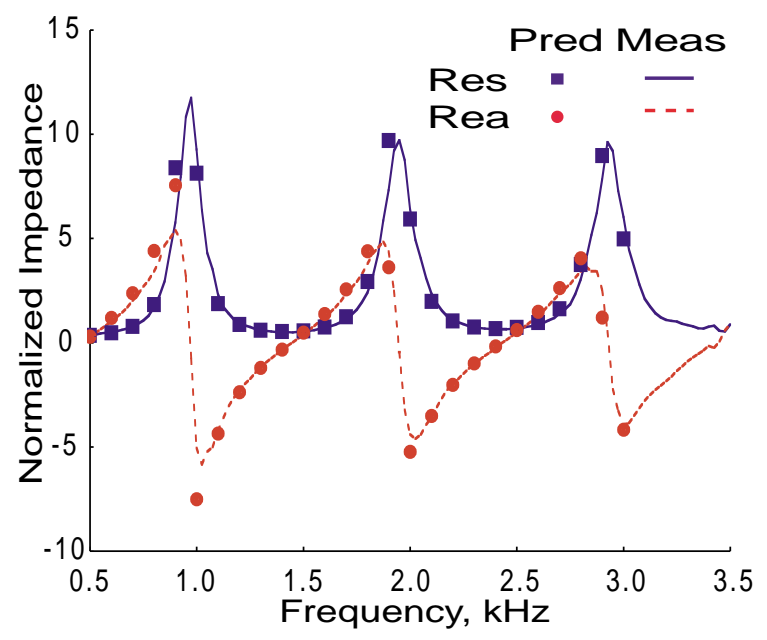

Figure 8. Normalized impedance for Configuration C2 (straight 20-channel, baseline - length, 2L).

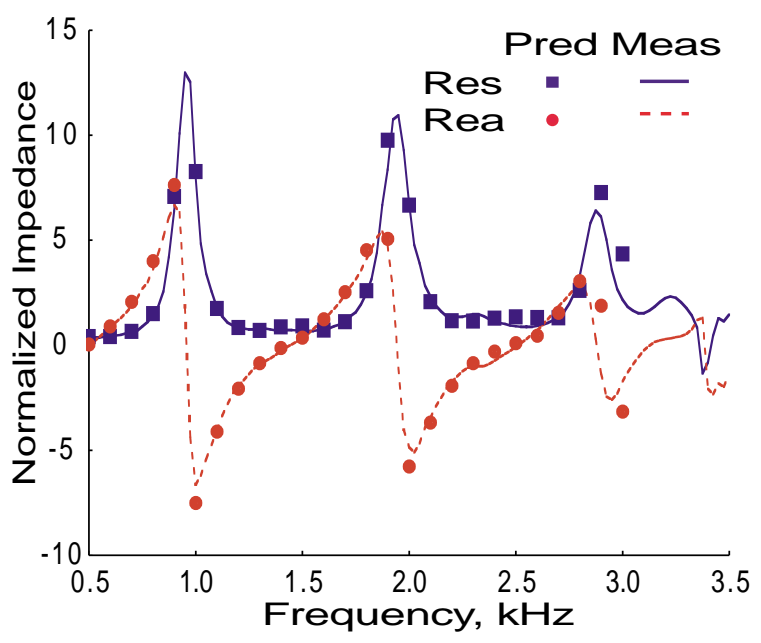

Figure 9. Normalized impedance for Configuration C3 (straight 17-channel \& 30-degree skew).

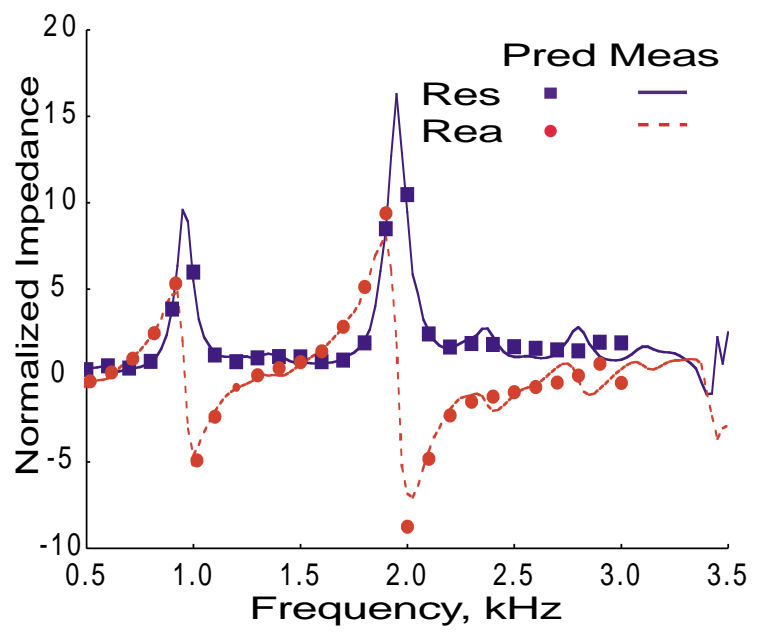

Figure 10. Normalized impedance for Configuration C4 (straight 17-channel \& 60-degree skew). 


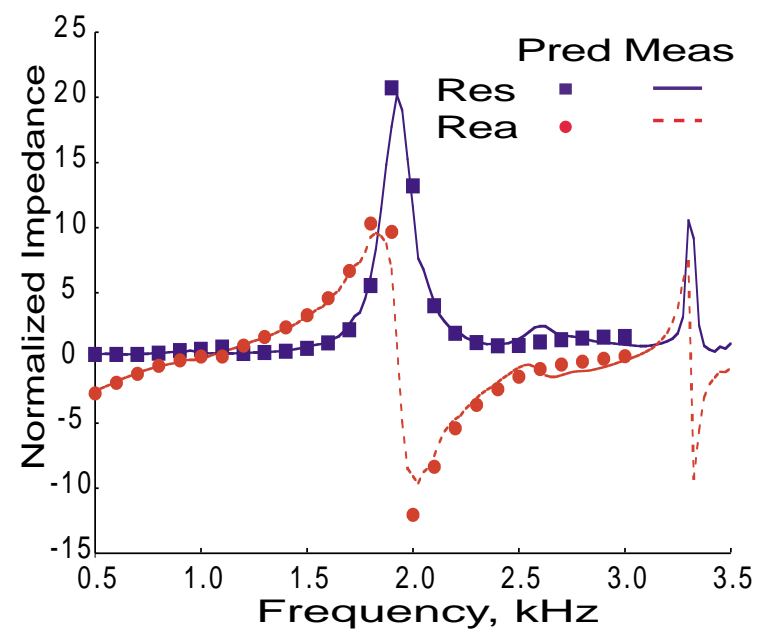

Figure 11. Normalized impedance for Configuration C5 (isolated 17-channel at 30 degree skew).

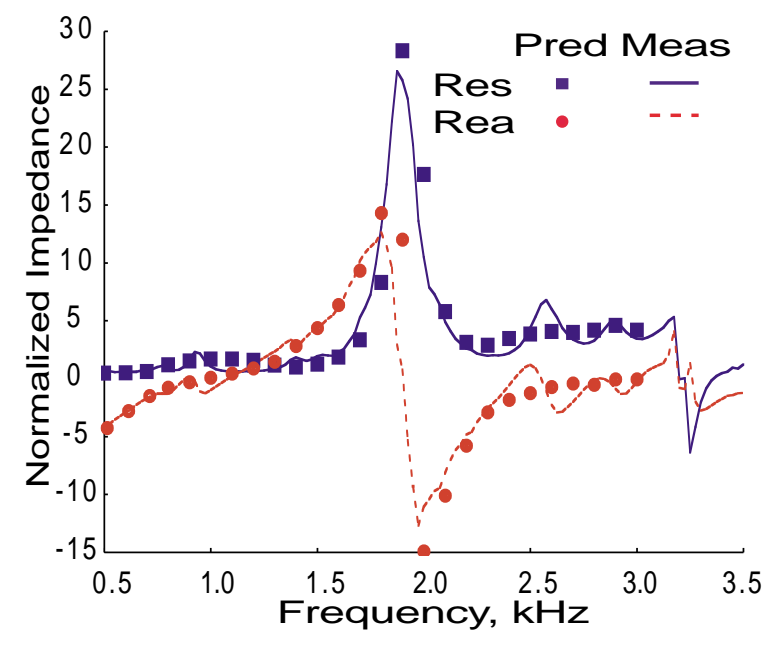

Figure 12. Normalized impedance for Configuration C6 (isolated 10-channel at 60-degree skew). 